\title{
Purpose-Dependent Consequences of Temporal Expectations Serving Perception and Action
}

\author{
${ }^{\circ}$ Freek van Ede, ${ }^{1,3}{ }^{\circledR}$ Gustavo Rohenkohl, ${ }^{1,2}{ }^{-}$Ian Gould, ${ }^{1,2}$ and ${ }^{\circledR}$ Anna C. Nobre ${ }^{1,2}$ \\ ${ }^{1}$ Oxford Centre for Human Brain Activity, Wellcome Centre for Integrative Neuroimaging, Department of Psychiatry, University of Oxford, Oxford \\ OX3 7JX, United Kingdom, ${ }^{2}$ Department of Experimental Psychology, University of Oxford, Oxford OX2 6GG, United Kingdom, and ${ }^{3}$ Institute for \\ Brain and Behavior Amsterdam, Department of Experimental and Applied Psychology, Vrije Universiteit Amsterdam, 1081BT, Amsterdam, The \\ Netherlands
}

Temporal expectations enable anticipatory brain states that prepare us for upcoming perception and action. We investigated the purpose-dependent nature and consequences of cued temporal expectations on brain and behavior in male and female human volunteers, using two matched visual-motor tasks that stressed either response speed or visual accuracy. We show that the consequences of temporal expectations are fundamentally purpose dependent. Temporal expectations predominantly affected response times when visual demands were low and speed was more important, but perceptual accuracy when visual demands were more challenging. Using magnetoencephalography, we further show how temporal expectations latch onto anticipatory neural states associated with concurrent spatial expectations-modulating task-specific anticipatory neural lateralization of oscillatory brain activity in a modality- and frequency-specific manner. By relating these brain states to behavior, we finally reveal how the behavioral relevance of such anticipatory brain states is similarly purpose dependent.

Key words: alpha oscillations; attention; behavioral performance; beta oscillations; task-dependent; temporal expectation

Significance Statement

Knowing when events may occur helps to prepare neural activity for upcoming perception and action. It is becoming increasingly clear that distinct sources of temporal expectations may facilitate performance via distinct mechanisms. Another relevant dimension to consider regards the distinct purposes that temporal expectations may serve. Here, we demonstrate that the consequences of temporal expectations on neurophysiological brain activity and behavior are fundamentally purpose dependent, and show how temporal expectations interact with task-relevant neural states in a modality- and frequency-specific manner. This brings the important insight that the ways in which temporal expectations influence brain and behavior, and how brain activity is related to behavior, are not fixed properties but rather depend on the task at hand.

Received May 10, 2020; revised Aug. 3, 2020; accepted Aug. 6, 2020.

Author contributions: G.R., I.G., and A.C.N. designed research; G.R., I.G., and A.C.N. performed research; F.v.E. analyzed data; F.v.E. and A.C.N. wrote the paper.

This research was funded by a Marie Skłodowska-Curie Fellowship from the European Commission (ACCESS2WM) to F.v.E., a Wellcome Trust Project Grant (089903) and Senior Investigator Award (104571/Z/ 14/Z), and a James S. McDonnell Foundation Understanding Human Cognition Collaborative Award (220020448) to A.C.N.; and by the National Institute for Health Research Oxford Health Biomedical Research Centre. The Wellcome Centre for Integrative Neuroimaging is supported by core funding from the Wellcome Trust (Grant 203139/Z/16/Z). The funders had no role in study design, data collection, and analysis; decision to publish; or preparation of the manuscript. We thank Clare Palmer, Jessica Gaunt, and Simone Heideman for help with data handling and data collection.

G. Rohenkohl's present addresses: Department of Physiology, Institute of Biosciences, University of São Paulo, 05508-090 São Paulo, Brazil; and Ernst Strüngmann Institute (ESI) for Neuroscience in Cooperation with Max Planck Society, 60528 Frankfurt, Germany.

I. Gould's present address: Saluda Medical Pty Ltd, Sydney 2064, New South Wales, Australia.

Correspondence should be addressed to Freek van Ede at freek.van.ede@vu.nl.

https://doi.org/10.1523/JNEUROSCl.1134-20.2020

Copyright @ 2020 van Ede et al.

This is an open-access article distributed under the terms of the Creative Commons Attribution License Creative Commons Attribution 4.0 International, which permits unrestricted use, distribution and reproduction in any medium provided that the original work is properly attributed.

\section{Introduction}

Temporal expectations help us prepare for upcoming perception (Jones and Boltz, 1989; Coull and Nobre, 1998; Ghose and Maunsell, 2002; Lange and Röder, 2006; Lakatos et al., 2008; Jaramillo and Zador, 2011; Lima et al., 2011; Rohenkohl et al., 2012; Vangkilde et al., 2012; Meijer et al., 2016; Auksztulewicz et al., 2018) as well as action (Riehle et al., 1997; Shin and Ivry, 2002; Janssen and Shadlen, 2005; Schoffelen et al., 2005; Praamstra et al., 2006; van Elswijk et al., 2007; Los et al., 2017; Heideman et al., 2018b, 2020; Boettcher et al., 2020). It is increasingly recognized that distinct sources of temporal expectations, such as associations, rhythms, hazard rates, and sequences (Nobre and van Ede, 2018), may facilitate performance via distinct neural mechanisms (Rohenkohl et al., 2011; Triviño et al., 2011; de la Rosa et al., 2012; Breska and Deouell, 2014; Morillon et al., 2016; Breska and Ivry, 2018; Nobre and van Ede, 2018; Bouwer et al., 2020). In addition to distinct sources of temporal expectation, another relevant dimension to consider regards the distinct purposes that temporal expectations may serve (Shalev 
et al., 2019). Is it possible for the same type of temporal expectation to have distinct consequences on brain activity and on behavioral performance depending on the anticipated task demands?

To address this, we used a classic associative temporal orienting task (Coull and Nobre, 1998; Nobre, 2001), in which cues predict the time at which a target will appear, and measured neural anticipation using magnetoencephalography (MEG). Critically, we varied whether the anticipated task stressed motor demands, by requiring a speeded response to an easy-to-discriminate visual target ("motor task"), or stressed perceptual demands, by requiring a difficult visual discrimination ("visual task"). In both tasks, cues also provided valid spatial foreknowledge about the relevant response hand (motor task) or visual target location (visual task). This allowed us to assess the influence of temporal expectations in the presence of concurrent spatial expectations (Doherty et al., 2005; Rohenkohl et al., 2014) and to ask whether temporal expectations modulate purpose-specific electrophysiological signatures of spatial anticipation in a modality- and frequency-specific manner.

\section{Materials and Methods}

Data from the visual task has previously been published in the context of an aging study where we compared these data to those from a group of older participants doing the same task (Heideman et al., 2018a). The central aspect of the current article involves the comparison between the visual task and the matched motor task in which the same participants took part. Tasks were matched in that we used identical spatial-temporal cues and cue validities in both tasks (while varying task demands between tasks). Neither the data from the current motor task nor their comparison to data from the visual task have been published previously.

\section{Participants}

Twenty right-handed human volunteers (7 female; age range, 18-33 years; mean age, 24.1 years) participated in both the motor task and the visual task. All participants had normal or corrected-to-normal vision. Participants gave informed consent before participation and received monetary compensation for their time. Experimental procedures were approved by the Central University Research Ethics Committee of Oxford. Data from all participants were retained for analysis.

\section{Tasks and procedures}

Tasks were programmed in Psychtoolbox (Brainard, 1997) in MATLAB. Stimuli were projected at a $60 \mathrm{~Hz}$ refresh rate onto a $58 \times 46 \mathrm{~cm}$ screen that was placed $\sim 120 \mathrm{~cm}$ in front of the participant, who performed the tasks seated in the chair of the MEG.

a

b

C
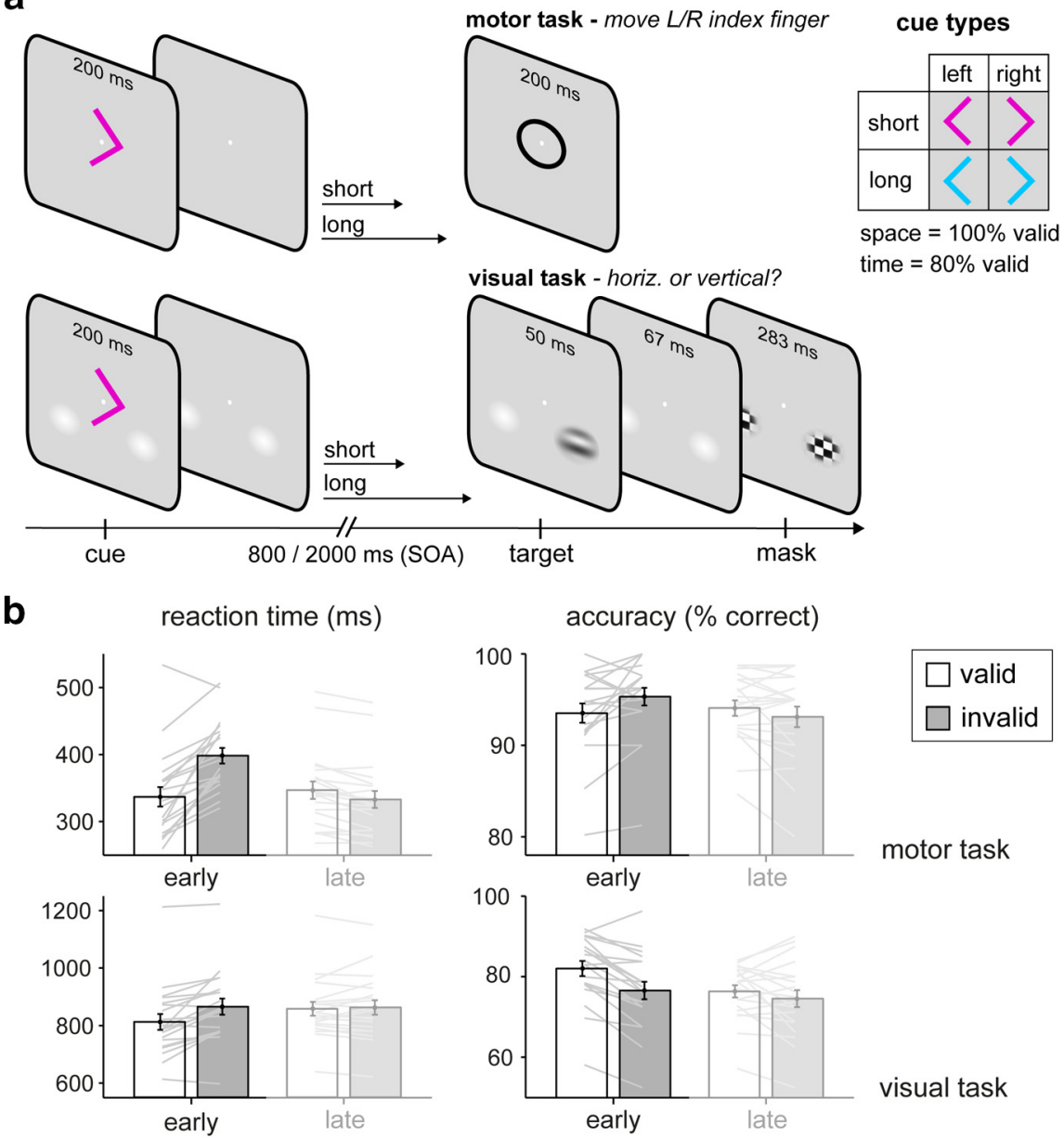

motor task

validity effect early targets $(\Delta \%)$
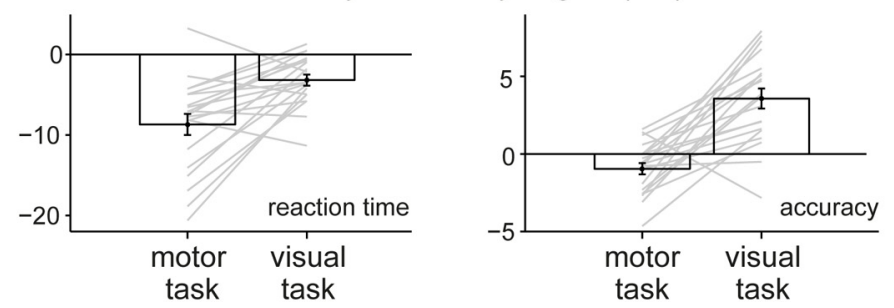

Figure 1. Spatial-temporal expectations benefit vision and action in distinct ways. $\boldsymbol{a}$, In the motor task, participants performed a speeded response to the central " 0 " target (or refrained from responding when the target was an " $\mathrm{x}$ " instead; $20 \%$ no-go trials). In the visual task, participants judged whether a lateralized visual target (masked by bilateral checkerboards) was horizontal or vertical. The same spatial-temporal cues (top right) preceded targets in both tasks. Cue direction instructed the relevant response hand (motor task) or predicted the side of the visual target (visual task) with 100\% validity. Cue color predicted target time with $80 \%$ validity. $\boldsymbol{b}$, Reaction times and the percentage of correct responses in the motor (top) and visual (bottom) tasks as a function of temporal cue validity. Performance to late targets is shown merely for completeness (see Materials and Methods). c, Temporal cue validity effects in both tasks, expressed as a percentage change between expected versus unexpected early targets. Error bars indicate \pm 1 SEM. Gray lines show individual participants.

Participants performed two versions of a cued spatial-temporal orienting task (Fig. 1a), referred to as the motor task and the visual task. Identical visual cues and cue validities (Fig. $1 a$, top right) were used in both tasks. Cues were presented centrally for $200 \mathrm{~ms}$ and were at a $2.18^{\circ}$ visual angle in width and height, with a linewidth of $0.1^{\circ}$. Cue direction (left or right) indicated with $100 \%$ validity what response hand was required (motor task) or on which side the visual target would occur (visual task). Cue color (blue or pink) predicted with $80 \%$ validity the time at which the target would be presented: after a stimulus-onset-asynchrony (SOA) of 800 or $2000 \mathrm{~ms}$. The same intervals were used in both tasks. The mapping of color to SOA was counterbalanced across participants but was kept consistent across both versions of the task. 
Participants were explicitly informed about color-to-interval mappings and familiarized themselves with these mappings in practice trials in which the cues were $100 \%$ valid. Trials began with the onset of a fixation dot $\left(0.88^{\circ}\right)$ that appeared after an intertrial interval of $1500 \pm 250 \mathrm{~ms}$ and that preceded cue onset by $750 \pm 250 \mathrm{~ms}$.

In both tasks, participants held their index fingers on two fiber-optic response boxes (custom made by the O.V. Lounasmaa Laboratory, Aalto University, Espoo, Finland) and responded by lifting the index finger of the left or right hand.

In the motor task, participants were instructed to lift the index finger of the cued hand as quickly as possible after a central "o" stimulus $\left(6.4^{\circ}\right.$ diameter) appeared around fixation. We inserted $20 \%$ of trials with an " $x$ " stimulus instead and instructed participants to refrain from responding in these trials (which they did successfully in a mean \pm SEM of $74.63 \pm 4.17 \%$ of trials). No-go trials were included to ensure that participants waited for the target to appear before making a response. In the motor task, target stimuli stayed on the screen for $200 \mathrm{~ms}$.

In the visual task, the target consisted of a horizontally or vertically tilted Gabor patch (diameter, $1.96^{\circ}$; spatial frequency, 2 cycles $/^{\circ}$ ) presented at the cued location to the left or right of fixation on top of one of two faint luminance pedestals ( $10 \%$ contrast). Pedestals and targets were anchored at $4.78^{\circ}$ below the horizontal meridian at a distance of $3.38^{\circ}$ from the vertical meridian. Target stimuli were presented for $50 \mathrm{~ms}$ and masked by bilateral checkerboard stimuli after an SOA of $117 \mathrm{~ms}$. Masks were presented for $283 \mathrm{~ms}$ and were created by applying a Gaussian vignette to the convolution of $100 \%$ square-wave gratings at the two possible target orientations. Participants were instructed to report as accurately and as quickly as possible whether the grating was horizontal or vertical using the left and right index fingers. Responses in this task, however, were not linked to the direction of the cue, but to the identity of the visual target (horizontal or vertical, which was varied independent of cue direction and, consequently, of target location). The mapping between target identity and response hand was counterbalanced across participants.

One week before the first MEG session, participants came in for a 90 min session that allowed us to staircase the difficulty of the visual task. First, they familiarized themselves with the task across five blocks of 120 trials, in which we gradually decreased the time of target presentation down to $50 \mathrm{~ms}$. We then applied an adaptive staircase procedure (as described in the study by Rohenkohl et al., 2014) in which we titrated the contrast of the Gabor patch contrast until discrimination was performed at $75 \%$ accuracy. During the staircase session, temporal cues were $100 \%$ predictive of the target time.

We counterbalanced the order of the visual task and motor task MEG sessions across participants. Each session contained 800 trials, distributed across four blocks of $\sim 15$ min each. Participants could take selfpaced breaks after every 40 trials. Participants came into the laboratory at separate days to complete each session, with sessions typically being $<1$ week apart, but never $>20 \mathrm{~d}$ apart.

\section{Analysis of behavioral data}

We considered the following two dependent variables in both tasks: reaction time (RT) and percentage of correct responses. Reaction times were considered only for trials in which a response was recorded in the 200-2000 ms response window (defined relative to target onset). For analysis of the motor task data, we included both go and no-go trials for calculating accuracy (but only go trials for reaction time). We obtained the same pattern of behavioral accuracy results and statistical inferences when only go-trials were included, unless explicitly specified otherwise in our Results section.

We decided a priori to focus our analysis on the most informative data. To this end, we exclusively compared performance to early targets when these were expected (valid) versus unexpected (invalid). In cued temporal orienting tasks, it is well established (Nobre, 2001; Nobre and van Ede, 2018) that temporal cueing benefits are much more profound for early than late targets (once the early interval passes, participants can update their expectations, washing out cue validity effects for late targets). Focusing on the early targets not only simplified our analyses and increased sensitivity, it also allowed for a better comparison to the MEG analyses which, for the same reason, focused on the early interval after the cue. For completeness and transparency, we show performance to late targets in our Results section.

Because our tasks were designed to vary in overall task demandsemphasizing either response speed or perceptual accuracy-we expected large differences in overall performance between tasks, which were not our main focus. Instead, we decided a priori to focus our main statistical analyses on the performance benefits conferred by valid temporal expectations (at the early interval). To quantify the relative benefits of temporal predictions in a manner that accounted for the large global differences in RT and accuracy between tasks, we expressed validity effects as the normalized percentage change of each dependent variable [i.e., $($ valid - invalid $) /($ valid + invalid $\left.){ }^{\star} 100\right]$. We thus used paired-samples $t$ tests as our primary statistical evaluation for comparing cue validity effects between tasks. For completeness, we also report the results from a repeated-measures ANOVA with the factors "task" and "validity," which also enabled us to formally evaluate the anticipated main effects of task.

As measures of effect size, we used Cohen's $d$ for all reported $t$ tests, and partial $\eta^{2}$ values for the ANOVA.

\section{MEG acquisition and analysis}

We acquired magnetoencephalography using a 306-channel VectorView MEG system (Elekta) that was housed in a magnetically shielded room at the Oxford Centre for Human Brain Activity. Data were acquired at a sampling rate of $1000 \mathrm{~Hz}$. Head position was continuously tracked using four head position indicator (HPI) coils, placed behind the ears and on the forehead. A horizontal and a vertical electro-oculogram (EOG) were concurrently acquired by placing four $\mathrm{Ag} / \mathrm{AgCl}$ electrodes surrounding the eyes.

MEG preprocessing. Data were analyzed in FieldTrip (Oostenveld et al., 2011). Before loading the data into FieldTrip, data were cleaned using the Neuromag MaxFilter software version 2.0. At this stage, data from all participants were aligned to a common spatial positioning using the continuous HPI data, and an independent component analysis (Bell and Sejnowski, 1995) was applied to remove components associated with eye movement and blinks as detected by the EOGs. All subsequent analyses in FieldTrip were performed on the 204 planar-gradiometer channels that we combined into 102 combined-planar channels using the singular value decomposition method. Trials with excessive variance were identified and removed following visual inspection using the function " $\mathrm{ft} \_$rejectvisual" with the summary method. After trial removal, mean ( \pm SEM) of $763 \pm 11$ (motor task) and $716 \pm 28$ (visual task) trials were retained for analysis.

Predefined channel selection. To increase the sensitivity of our analyses, we focused our analyses on data in predefined clusters of left and right posterior (visual) and central (motor) MEG channels. Channels were chosen based on prior MEG studies from our laboratory that have consistently implicated the same set of posterior and central channels for capturing neural activity related to lateralized manual actions and lateralized visual stimuli (Heideman et al., 2018a,b, 2020). Specifically, we included the following four (combined) channels in each cluster: left central (motor): \{'MEG0412 + 0413','MEG0422 + 0423','MEG0432 + 0433','MEG0442 + 0443'\}; right central (motor): \{'MEG1112 + 1113', 'MEG1122 + 1123','MEG1132 + 1133','MEG1142 + 1143'\}; left posterior (visual): \{'MEG1912 + 1913','MEG1922 + 1923','MEG1942 + 1943', 'MEG2042 + 2043'\}; and right posterior (visual): \{'MEG2032 + 2033', 'MEG2312 + 2313','MEG2322 + 2323','MEG2342 + 2343'\}.

While we selected these channel clusters based on independent data, we could confirm their validity and appropriateness in the current data (Fig. $2 a$, overlay of these channel clusters on the relevant left vs right topographies). For the analysis of nonlateralized visual and motor activity, we included one additional posterior channel that was not part of the left/right channel clusters because it was a midline channel: $\{$ 'MEG2112 + 2113'\}.

Spectral analyses. We calculated spectral power using a short-time Fourier transform with a sliding time window of $300 \mathrm{~ms}$ on Hanningtapered data. The analysis window was advanced over the data in steps of $10 \mathrm{~ms}$. Power was calculated for frequencies between 3 and $40 \mathrm{~Hz}$ in steps of $0.5 \mathrm{~Hz}$. Power values were contrasted between conditions and 
were expressed as a percentage change: ((condition $\mathrm{A}-$ condition $\mathrm{B}) /$ (condition $\mathrm{A}+$ condition $\mathrm{B}))^{\star} 100$. To depict the topographies associated with relevant condition comparisons (i.e., left vs right; short vs long), we focused on the predefined $8-12 \mathrm{~Hz}$ (alpha) and $13-30 \mathrm{~Hz}$ (beta) frequency bands in the predefined $400-800 \mathrm{~ms}$ anticipatory (i.e., pretarget) window. For the comparison between contralateral and ipsilateral power, we compared activity following left- and right-directing cues, separately for left and right channel clusters, and subsequently pooled the contralateral versus ipsilateral contrasts between them. For our main analysis, we used the predefined central (motor) channels to calculate contralateral versus ipsilateral contrasts in the motor task, and the predefined posterior (visual) channels to calculate contralateral versus ipsilateral contrasts in the visual task.

Statistical analyses of MEG data. Statistical analyses focused on contrasted time-frequency maps extracted over the predefined central (motor) and posterior (visual) channel clusters; as well as on extracted time courses collapsed over the predefined $8-12 \mathrm{~Hz}$ alpha band (visual task lateralization data) and $13-30 \mathrm{~Hz}$ beta band (motor task lateralization data). To bypass the multiple-comparisons problem, we used a nonparametric cluster-based permutation approach (Maris and Oostenveld, 2007), and used the Fieldtrip default cluster settings with 10,000 permutations. The majority of our cluster-based permutation tests clustered over the time dimension using predefined frequency bands and channels. In some cases, however, cluster-based permutation tests clustered over both time and frequency. We compared time courses of spatial lateralization following short and long cues, as well as for conditions separated by performance to early targets. Performance sorting for reaction time was performed using a median split within each participant. We focused all MEG analyses on the early interval after the cue where temporal expectation effects are known to be most pronounced (see Analysis of behavioral data, above). For the motor task brain behavior analysis sorted by accuracy, 2 (of 20) participants had an insufficient number of incorrect trials $(<5$ incorrect trials) to be included (compared with $25.06 \pm 4$ incorrect trials/participant in the remaining sample of 18 participants).

\section{Results}

Twenty healthy human volunteers participated in two spatialtemporal anticipation tasks (Fig. 1a) while we recorded their brain activity using MEG. The two tasks were performed during separate visits in counterbalanced order. In the motor task, participants responded as quickly as possible to the appearance of a central "o" stimulus. In the visual task, participants discriminated whether the tilt of a masked visual stimulus was horizontal or vertical. Cues were identical in the two tasks and enabled anticipation in space and time (Fig. $1 a$, right). Cue direction informed with $100 \%$ validity that the response hand (motor task) or visual stimulus (visual task) would be left or right. Cue color informed whether the target would most likely ( $80 \%$ valid) appear after a short or a long cue-target interval (800 or $2000 \mathrm{~ms}$ after cue onset).

\section{Spatial-temporal expectations benefit vision and action in distinct ways}

We first ascertained that participants used the temporal cue information to their benefit in both tasks. Figure $1 b$ shows performance in the motor (top) and visual (bottom) tasks, as a function of whether targets occurred at the cued time (valid) or not (invalid). Focusing on early targets-where temporal cueing effects are known to be captured most sensitively (Nobre, 2001; Nobre and van Ede, 2018) - we found that valid temporal expectations yielded significantly faster responses (Fig. $1 b$, left) in both the motor task $\left(t_{(19)}=-6.457, p=3.448 \mathrm{e}-6, d=-1.444\right)$ and the visual task $\left(t_{(19)}=-4.576, p=2.061 \mathrm{e}-4, d=-1.023\right)$. For accuracy (Fig. 1b, right), valid temporal expectations also improved performance for early targets on the visual task $\left(t_{(19)}=4.990\right.$, $p=1.128 \mathrm{e}-5, d=1.116)$ but made participants slightly worse in the motor task $\left(t_{(19)}=-2.541, p=0.019, d=-0.568\right)$, possibly reflecting a shift in the speed-accuracy trade-off in this task where overall accuracy was very high. This reduction in accuracy in the motor task was driven by a larger number of responses to valid versus invalid early no-go targets $\left(t_{(19)}=4.236, p=4.467 \mathrm{e}\right.$ $4, d=0.947$ ); when only considering early go targets, valid temporal expectations were associated with better performance $\left(t_{(19)}=2.216, p=0.039, d=0.496\right)$.

For completeness, we also ran an ANOVA with the factors cue validity and task (again focusing on data from early targets). This confirmed a significant main effect of cue validity on both RT $\left(F_{(1,19)}=47.79, p=1.365 \mathrm{e}-6, \eta_{p}^{2}=0.797\right)$ and accuracy $\left(F_{(1,19)}=9.537, p=0.006, \eta_{p}^{2}=0.275\right)$. However, this also showed robust main effects of task on both dependent variables, whereby responses in the motor task were generally much faster $\left(F_{(1,19)}=\right.$ 279.288, $\left.p=8.107 \mathrm{e}-13, \eta_{p}^{2}=0.996\right)$ and more accurate $\left(F_{(1,19)}=\right.$ $\left.68.452, p=1.013 \mathrm{e}-7, \eta_{p}^{2}=0.968\right)$ than in the visual task. To deal with these vast differences in average RT and accuracy between tasks, we therefore expressed the validity effect in each task as a relative (percentage) change before comparing validity effects between tasks, to which we turn next (Fig. 1c).

In the two tasks, the same participants viewed the same cues followed by the same intervals and target probabilities; thus enabling equivalent degrees of temporal anticipation. Yet, we observed remarkably distinct patterns of performance benefits between tasks (Fig. 1c). Temporal expectations conferred significantly larger reaction time benefits to early targets in the motor task than in the visual task [Fig. $1 c$, left; $8.69 \%$ (motor) vs $3.17 \%$ (visual) faster responses; $t_{(19)}=-4.143, p=5.525 \mathrm{e}-4, d=$ $-0.926]$, but significantly larger accuracy benefits to early targets in the visual task than in the motor task [Fig. 1c, right; $-0.87 \%$ (motor) versus $3.34 \%$ (visual) more accurate responses; $t_{(19)}=$ $-5.441, p=2.998 \mathrm{e}-5, d=-1.217$; and this was also the case when only including early go trials for the motor task; $t_{(19)}=$ $-3.752, p=0.001, d=-0.839]$.

Thus, the nature of the benefits of temporal foreknowledge on performance are purpose dependent: affecting predominantly reaction time when perceptual demands are low and speed is of the essence (motor task), and, conversely, accuracy when the perceptual demands are high and response speed is less important (visual task).

\section{Temporal expectations latch onto modality-specific substrates of spatial anticipation}

In both tasks, temporal expectations occurred alongside of valid foreknowledge about space-whether the response was required with the left or right hand (motor task) or whether the visual target would appear left or right on the screen (visual task).

To characterize the neural dynamics of spatial anticipation in our tasks, we collapsed over short and long cues and contrasted neural activity following left- versus right-directing cues. This confirmed clear lateralization of neural activity in the $8-12 \mathrm{~Hz}$ alpha band and the $13-30 \mathrm{~Hz}$ beta band in both tasks (Fig. 2a), which is consistent with many prior studies (Pfurtscheller and Berghold, 1989; Worden et al., 2000; Schoffelen et al., 2005; Thut et al., 2006; Donner et al., 2009; Jensen and Mazaheri, 2010; Gould et al., 2011, 2012; van Ede et al., 2011; Kizuk and Mathewson, 2017; Heideman et al., 2018a,b, 2020; Boettcher et al., 2020). As expected, the topographical distribution and spectral content of these spatial anticipation signatures were distinct between tasks. In the motor task, the lateralization was most 


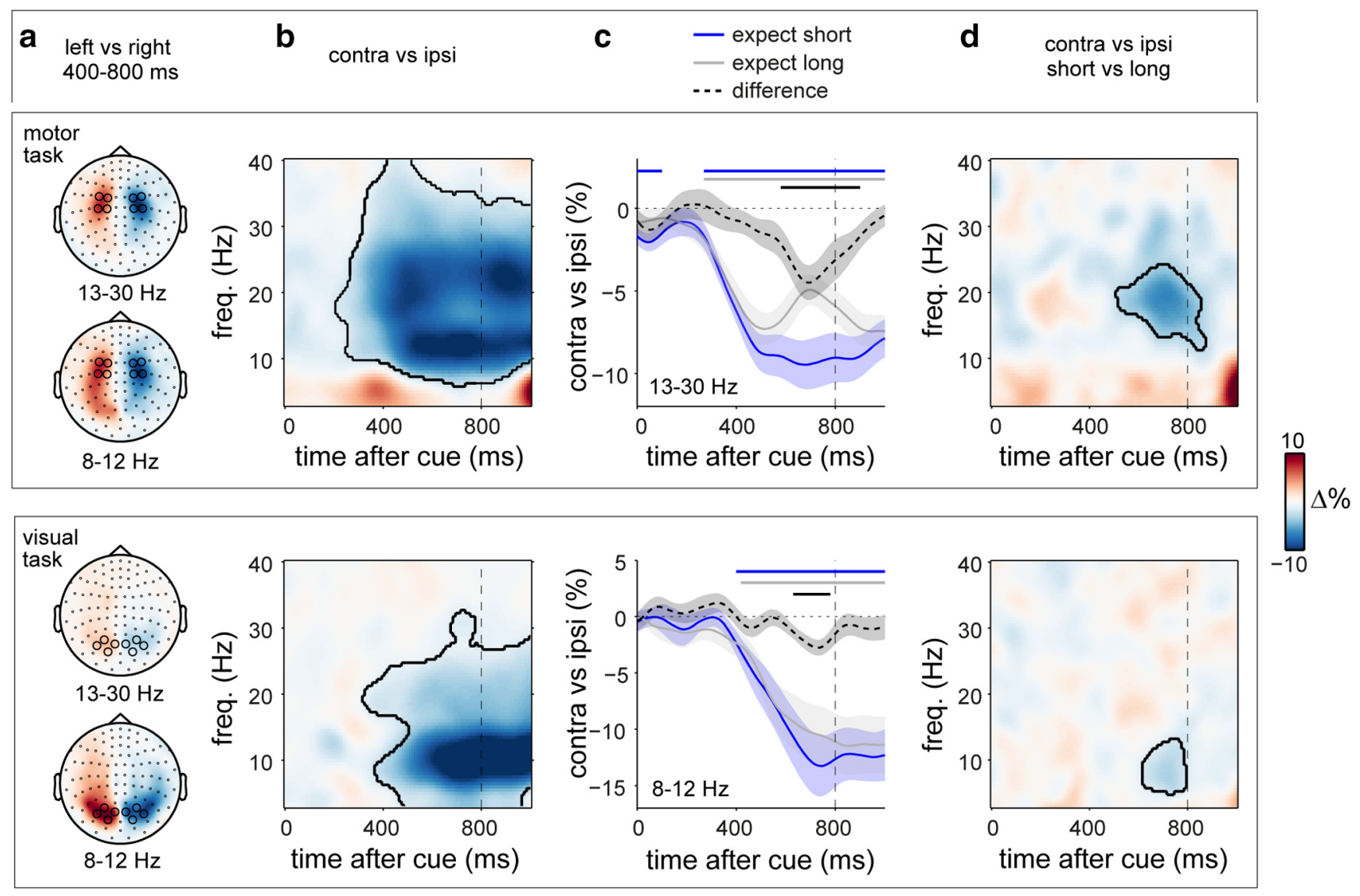

Figure 2. Temporal expectations latch onto modality- and frequency-specific substrates of spatial anticipation. $\boldsymbol{a}$, Topographies of power differences following left- versus right-directing cues in the 8-12 Hz alpha bands and 13-30 Hz beta bands, separately for the motor (top) and visual (bottom) tasks (collapsed over short and long cues). $\boldsymbol{b}$, Time-frequency plots of the neural lateralization in $\boldsymbol{a}$, showing the difference in spectral power contralateral versus ipsilateral to the cued side, extracted over the predefined left and right channel clusters depicted in $\boldsymbol{a}$. Black outlines indicate significant clusters following permutation analyses (Maris and Oostenveld, 2007). $\boldsymbol{c}$, Time courses of neural lateralization in $\boldsymbol{b}$, separated by temporal expectation conditions. Shadings indicate \pm 1 SEM. Horizontal lines indicate significant clusters. $\boldsymbol{d}$, Time-frequency representation of the difference in neural lateralization when the target was expected early versus late. Vertical dashed lines indicate the time at which early targets would occur.

pronounced in central (putative motor) MEG channels, and the neural modulation was pronounced across the $8-30 \mathrm{~Hz}$ band (Fig. $2 a, b$, top row; cluster, $p<0.0001$ ). In contrast, in the visual task the lateralization was most pronounced in posterior (putative visual) MEG channels, and was particularly pronounced in the $8-12 \mathrm{~Hz}$ alpha band (Fig. $2 a, b$, bottom row; cluster, $p<0.0001$; Fig. 3).

Critically, these signatures of spatial anticipation were each sensitive to concurrent temporal expectations (Fig. 2c). In the motor task, the attenuation of beta activity in contralateral (vs ipsilateral) motor electrodes was significantly stronger in the early interval after the cue, if participants expected the target to occur early (Fig. $2 c$, top row; cluster, $p=0.0034$ ). Likewise, in the visual task, the contralateral attenuation of posterior alpha activity was significantly stronger in the early interval after the cue, if participants expected the visual target to occur early (Fig. $2 c$, bottom row; cluster, $p=0.0123$ ). A time- and frequency-resolved plot of the difference in spatial anticipation following short versus long cues (Fig. 2d) confirmed the spectral specificity of these spatialtemporal anticipation effects.

To assess the spatial and spectral specificity of these purposedependent spatial-temporal neural modulations, we additionally considered, separately for each task, alpha modulations in posterior channels (Fig. $3 a$, left), as well as alpha and beta modulations in central channels (Fig. $3 a$, right and middle). A direct comparison between tasks in these spatial-temporal modulations (Fig. 3b) revealed a significantly stronger spatial-temporal modulation of central beta activity for the motor versus the visual task (Fig. $3 b$, right; cluster, $p=0.006)$. In contrast, the spatial-temporal modulation of posterior alpha oscillations-while significant in the visual task, but not in the motor task (Fig. 3a, left)-did not survive the direct task comparison (Fig. 3b, left). This is likely because of the observation that a trend for a similar spatial-temporal modulation of posterior alpha oscillations was observed in the motor task (possibly reflecting visual imagery of using the left vs right hand). Moreover, in contrast to the highly robust spatial-temporal modulation of beta activity in the motor task, the spatial-temporal modulation of posterior alpha lateralization in the visual task was less pronounced overall.

Thus, in the presence of spatial foreknowledge, temporal expectations latch onto and modulate neural signatures of spatial anticipation, in a modality- and frequency-dependent manner. This was particularly clear for the central beta modulation, which was only observed (and significantly stronger) in the motor task.

\section{Anticipatory modulations covary with speed in the motor task but accuracy in the visual task}

Separating the data by performance to the early targets also revealed how the anticipatory modulation of central beta oscillations in the motor task was associated with faster responses (Fig. $4 a$, top; cluster, $p$ values $=0.0042,0.0186$ ) but had no systematic 
relation to response accuracy (Fig. $4 b$, top). While we noted how incorrect trials were associated with an initial increase in contralateral versus ipsilateral beta activity (i.e., early lateralization in the "wrong" direction; Fig. $4 b$, top), this effect did not survive clusterbased permutation testing and possibly reflects noise, because of the limited number of incorrect trials in the motor task.

In contrast, the anticipatory modulation of posterior alpha oscillations in the visual task was associated with more accurate perceptual discrimination (Fig. 4b, bottom; cluster, $p=$ 0.0137 ), but showed no systematic relation to response times (Fig. $4 a$, bottom). This is consistent with the observation that the cues primarily affected reaction time in the motor task, but accuracy in the visual task (Fig. 1c); and shows that such brainbehavior associations too, may be fundamentally purpose dependent.

\section{Temporal orienting for action is additionally associated with posterior alpha attenuation}

We set up our tasks such that demands were largest for action in the motor task and largest for vision in the visual task. At the same time, both were "visual-motor" tasks with visual targets and manual responses. We therefore also looked for general temporal orienting effects (collapsed over left/right spatial expectations) in posterior-visual and central-motor channels in both tasks. As shown in Figure 5, this revealed particularly clear temporal expectation modulations in the motor task (Fig. 5, top). Despite the fact that the visual "target" in the motor task was always clearly visible and easy to discriminate, we found a robust attenuation of alpha activity in the posterior MEG channels following cues predicting a short versus long interval (Fig. 5b, top; cluster, $p=0.0089$ ). This effect in the $8-12 \mathrm{~Hz}$ alpha band had a clear posterior topography (Fig. $5 a, 8-12 \mathrm{~Hz}$ topography in motor task) concentrating in the same channels that also showed the clearest alpha lateralization in the visual task (Fig. 2a). A similar, albeit weaker, posterior modulation appeared in the visual task (Fig. 5b, middle), though no clusters survived statistical evaluation. When collapsing across tasks to look at the main effect of temporal expectation, this posterior alpha modulation did survive (Fig. $5 b$, bottom; cluster, $p=0.015$ ).

In both tasks, we also observed similar alpha/beta attenuation in central sites (Fig. $5 c$; motor task cluster, $p=0.0044$; visual task cluster, $p=0.0158)$. This became particularly clear when looking at the main effect of temporal expectation, collapsed across tasks (Fig. $5 c$, bottom row; cluster, $p=0.008$ ). For the motor task, it is possible that this central modulation may directly reflect the
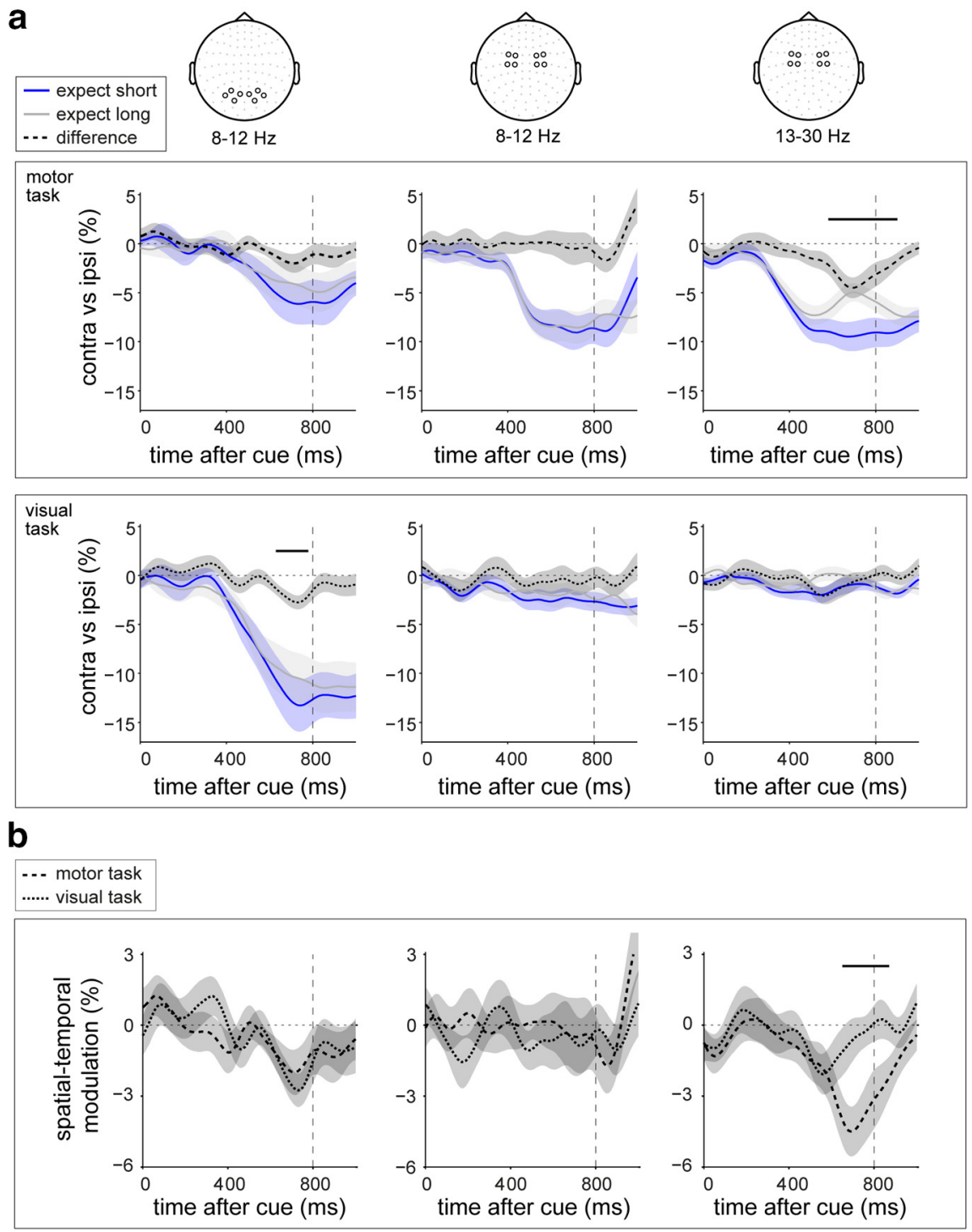

Figure 3. Spatial and spectral specificity of spatial-temporal neural modulations for vision and action. $\boldsymbol{a}$, Time courses of neural lateralization in posterior $8-12 \mathrm{~Hz}$ activity (left), central $8-12 \mathrm{~Hz}$ activity (center), and central $13-30 \mathrm{~Hz}$ activity (right) in the indicate \pm 1 SEM. Black horizontal lines indicate significant clusters of the modulation in neural lateralization by temporal expectation $(\boldsymbol{a})$ or their difference between tasks $(\boldsymbol{b})$. Top right and middle left panels are identical to those in Figure $2 c$.

contralateral beta attenuation associated with spatial-temporal expectations (Figs. 2, 3). However, the fact that a similar modulation appears in the visual task (where we did not see any spatialtemporal modulation in central channels) opens the possibility that these global modulations of temporal expectation may occur on top of the spatially specific signatures reported in the preceding figures.

\section{Discussion}

We investigated the behavioral consequences and anticipatory neural dynamics of cued spatial-temporal expectations in service of distinct task demands. This revealed how identical temporal cues can have drastically different consequences on performance - and be mediated by distinct neural modulations-when the task requires a speeded response versus a demanding perceptual judgment. The modulatory consequences of temporal expectation on neural activity and behavior thus not only depend on the 
a

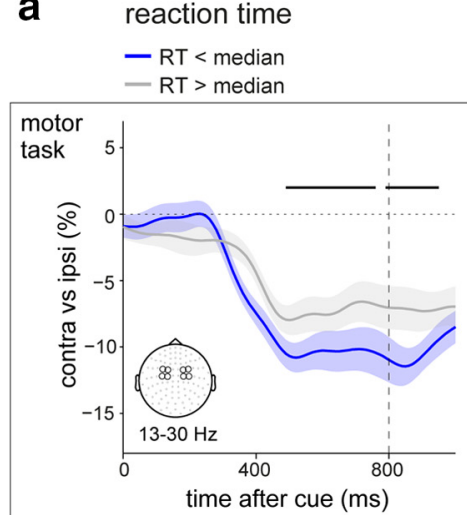

b

accuracy

- correct - incorrect
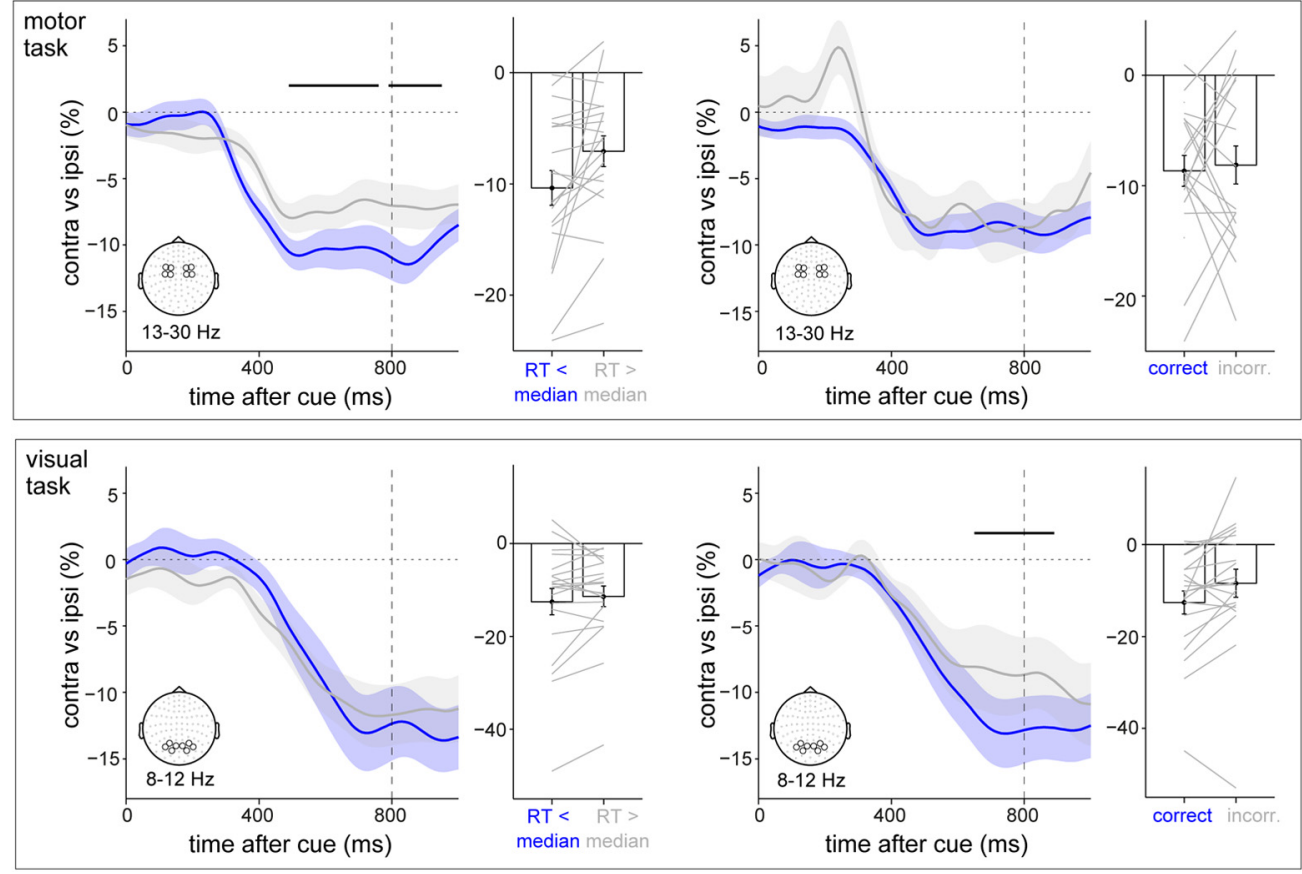

Figure 4. Anticipatory modulations predict speed in the motor task but accuracy in the visual task. $\boldsymbol{a}, \boldsymbol{b}$, Neural lateralization in central channels in the motor task and posterior channels in the visual task (compare Fig. $2 c$ ) separated by reaction times $(\boldsymbol{a})$ or accuracy $(\boldsymbol{b})$. Only trials with an early target were considered. Horizontal black lines indicate significant clusters of the difference between trials with "good" and "bad" performance. Bar graphs show the lateralization extracted over the significant windows in Figure $2 c$ (black horizontal lines) and serve primarily to depict the participant distribution of the effects. Gray lines show individual participants. For the accuracy-sorted data in the motor task, 2 (of 20) participants had insufficient incorrect trials to be included. Shadings and error bars indicate \pm 1 SEM.

source of temporal expectations, but also on its anticipated purpose (as also discussed in Shalev et al., 2019).

Our results provide a compelling reason to abandon attempts to pin down "the" way in which temporal expectations facilitate performance. In the past, numerous studies asked whether temporal expectations modulated early sensory stages of visual processing or were confined to modulate only late, response-related processes (Miniussi et al., 1999; Griffin et al., 2002; Correa et al., 2006; Lange et al., 2006; Lange and Röder, 2006). By equating the appearance and temporal probabilities of informative cues and manipulating the perceptual versus motor nature of perceptual demands, we show that temporal expectations operate in a contextually relevant manner by latching onto and optimizing other anticipatory biases afforded within a task, and predominantly affecting performance variables that are most relevant to the task. To demonstrate this, in our study, we kept the nature of temporal expectations fixed, using associative temporal cues, and manipulated only the task demands. Further flexibility in the modulatory consequences of temporal expectations have been proposed when different sources of expectations are compared (e.g., associative vs rhythmic; Rohenkohl and Nobre, 2011; Triviño et al., 2011; de la Rosa et al., 2012; Breska and Deouell, 2014; Morillon et al., 2016; Breska and Ivry, 2018; Nobre and van Ede, 2018; Bouwer et al., 2020).

In the current work, we focused on the modulatory consequences of temporal expectation (on both neural activity and on behavioral performance). As such, our data leave open the question of whether the origins of temporal expectations are purpose dependent as well. It remains a theoretical possibility that common sources of temporal expectation yield distinct modulatory consequences as a function of (anticipated) task demands.

By studying temporal expectations in the context of concurrent spatial expectations, our MEG data demonstrated how temporal expectations can latch onto existing neural modulations in a modality- and frequency-dependent manner (but see Fischer et al., 2013; Pomper et al., 2015), which was particularly clear for the spatial-temporal modulation of central beta activity that was observed exclusively in the motor task. These results are consistent with several prior demonstrations of such spatial-temporal modulation of oscillatory brain activity in service of perception (Rihs et al., 2009; Lima et al., 2011; van Ede et al., 2011; Heideman et al., 2020) and action (Schoffelen et al., 2005; Heideman et al., 2018b, 2020), including within the context of working memory (van Ede et al., 2017; Boettcher et al., 2020). Building on these studies, which targeted a single goal in isolation, the current study demonstrates the purpose-dependent manifestation of such spatial-temporal expectations within a single experiment, using the same cues and the same participant sample across the two tasks.

Action anticipation of the left/right response hand was associated with a spatially specific attenuation of activity that spanned the alpha and beta bands (Pfurtscheller and Berghold, 1989; McFarland et al., 2000; Donner et al., 2009; Boettcher et al., 2020). Yet, in the same motor task, the temporal modulation of this spatial anticipation signature appeared confined to the beta band. Thus, while sensorimotor alpha and beta activity may both contribute to effector-specific action preparation, beta activity appears particularly susceptible to time (consistent with more pronounced temporal modulation of anticipatory beta attenuation; van 
Ede et al., 2011; Todorovic et al., 2015). The absence of the spatial-temporal alpha modulation is unlikely to be accounted for by differences in the temporal sensitivity of alpha oscillations or our analyses hereof. For example, we observed timed modulation of alpha lateralization in the visual task (see also Rohenkohl and Nobre, 2011; Zanto et al., 2011; van Ede et al., 2017). Moreover, next to the central modulation in lateralized beta activity, in the same motor task we observed a pronounced and spatially global modulation of posterior alpha activity. However, unlike the spatial-temporal beta modulation in central sites, we propose that this posterior alpha modulation may not relate to motor preparation directly but may, instead, relate either to visual anticipation of the visual go signal in this task, though another possibility is that this more global signature reflects the engagement of parietal cortices associated with the control of attention.

It is tempting to attribute the spatial-temporal alpha versus beta modulations to anticipation of perception versus action, respectively. However, similar beta attenuation was previously documented during spatial-temporal somatosensory anticipation (van Ede et al., 2011). It may thus be more appropriate to attribute the frequencyspecific nature of the spatial-temporal anticipation signatures to the respective brain areas/networks involved (visual/parietal vs sensorimotor), rather than to a hard distinction between perception and action per se.

Our data also show that the relation between anticipatory neural modulations and performance (for review, see Ergenoglu et al., 2004; Thut et al., 2006; Hanslmayr et al., 2007; van Dijk et al., 2008; Mazaheri et al., 2009; Gould et al., 2011; Haegens et al., 2011; van Ede et al., 2012a,b, 2017) are contingent on task demands too. In our data, the degree of sensorimotor beta attenuation in the motor task predicted reaction times (but not accuracy; though we note that there were very few incorrect trials available in this task), while the degree of posterior alpha attenuation in the visual task predicted accuracy (but not reaction times). In the domain of perception, most previous reports have linked posterior alpha oscillations to measures of detection performance (hit rate) and reaction times. On this basis, it has been suggested that such "states" of attenuated alpha activity may primarily be associated with enhanced cortical excitability and thereby make you more likely to see a stimulus (criterion shift), without affecting the quality or accuracy of perception (Iemi et al., 2017; Benwell et al., 2018). We used a demanding backwardmasked two-alternative discrimination task and show that, in such a demanding perceptual discrimination task, alpha can be

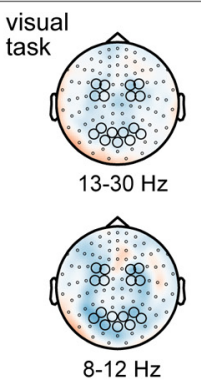

both

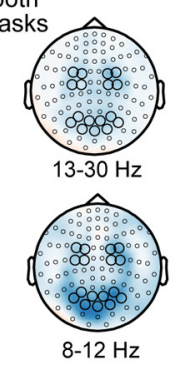

b
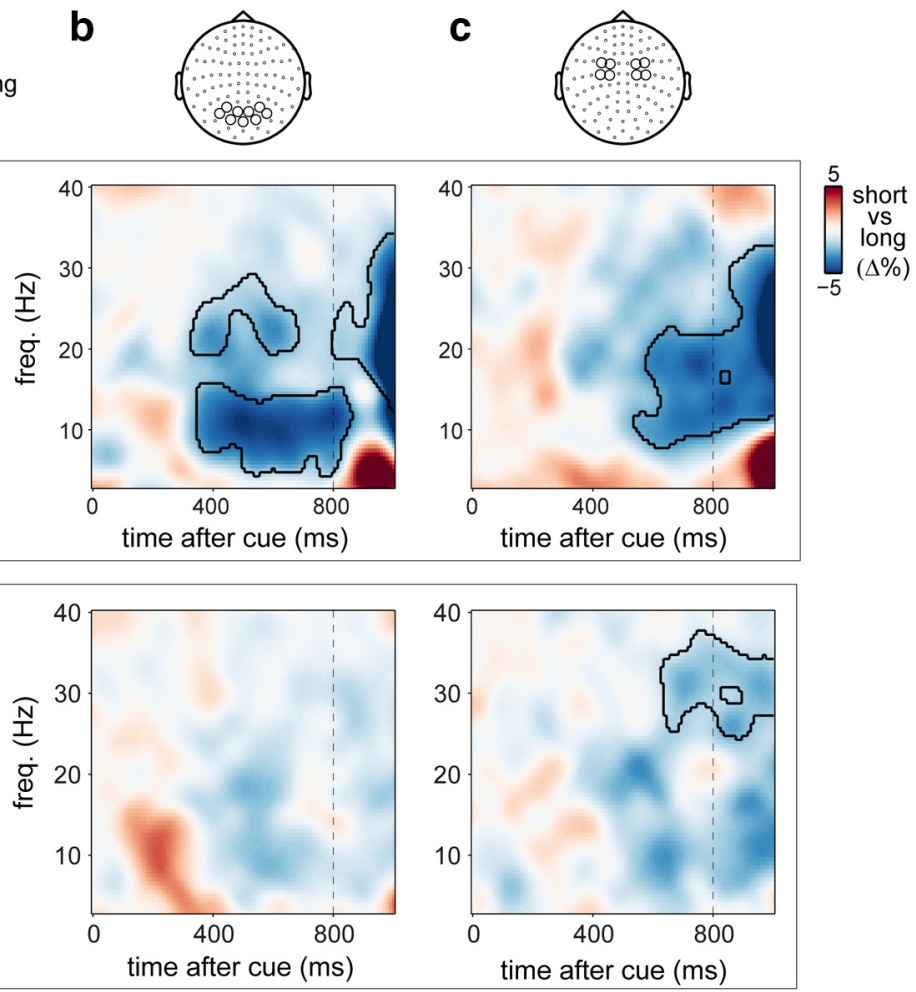

C
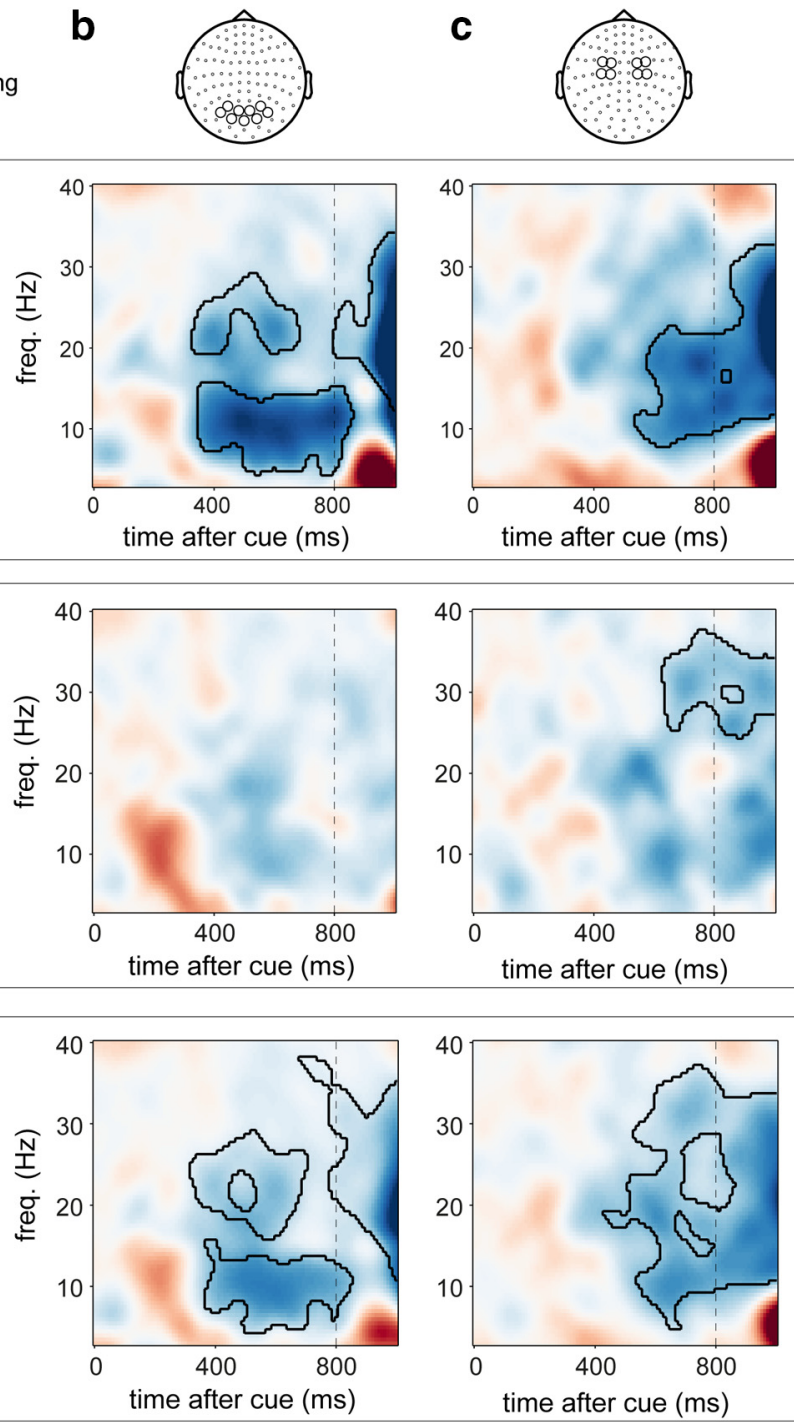

Figure 5. Global temporal expectation effects in posterior and central channels across both tasks. $\boldsymbol{a}$, Topographies of the and visual (middle) tasks (collapsed over left and right cues), as well as the main effect of temporal expectation collapsed across motor (c) channels in both tasks. Black outlines indicate significant clusters.

related (be it in a correlational sense) to perceptual accuracy. These brain-behavior associations are likely to reflect (at least in part) variability in the use of temporal expectations.

Complementing the temporal expectation-dependent amplification of spatial anticipation; we also found evidence for more global effects of temporal expectation. Most strikingly, we observed a robust posterior alpha attenuation in the motor task. While this likely reflects visual anticipation of the target in this task, this modulation is nevertheless remarkable as the visual target was very easy to see and discriminate. It does, however, beg the following question: why was this posterior modulation even more apparent in the motor task than in the visual task, in which visual demands were much higher? Possibly, this global modulation may be stronger when anticipating a central foveal visual target, which was the case only in our motor task.

Another possibility, however, is that there is greater motivation for relying on temporal expectations in the speeded response task, which injected urgency for completing sensorimotor processing as rapidly as possible. This would, in turn, lead to more 
profound neural modulations. In line with this, like the global posterior modulation, the identified spatial-temporal modulation also appeared more profound in the motor task (in central sites) than the corresponding spatial-temporal modulation in the visual task (in posterior sites). Such a purpose-dependent incentive to use temporal foreknowledge remains an interesting possibility for further investigation.

In our experiment, response speed was most important in the motor task, while accuracy was most important in the visual task. We do not intend to claim that motor anticipation will only ever influence response speed while visual anticipation will only ever influence accuracy-and likewise that their neural correlates are only relevant for either measure of performance. Instead, we emphasize that modulatory consequences may inevitably depend on task demands. Our tasks were set up to emphasize speed or accuracy in motor and visual tasks, respectively. In future work, complementary tasks could be devised that would emphasize accuracy in the motor task, but speed in the visual task. In such cases, the consequences of temporal expectations-and their neural modulations - on speed and accuracy may well reverse in their relation to perception and action.

\section{References}

Auksztulewicz R, Schwiedrzik CM, Thesen T, Doyle W, Devinsky O, Nobre AC, Schroeder CE, Friston KJ, Melloni L (2018) Not all predictions are equal: "what" and "when" predictions modulate activity in auditory cortex through different mechanisms. J Neurosci 38:8680-8693.

Bell AJ, Sejnowski TJ (1995) An information-maximization approach to blind separation and blind deconvolution. Neural Comput 7:1129-1159.

Benwell CSY, Keitel C, Harvey M, Gross J, Thut G (2018) Trial-by-trial covariation of pre-stimulus EEG alpha power and visuospatial bias reflects a mixture of stochastic and deterministic effects. Eur J Neurosci 48:25662584.

Boettcher SEP, Gresch D, Nobre AC, van Ede F (2020) Output planning at the input stage: action imprinting for future memory-guided behaviour. Available at SSRN: https://ssrn.com/abstract $=3550240$.

Bouwer FL, Honing H, Slagter HA (2020) Beat-based and memory-based temporal expectations in rhythm: similar perceptual effects, different underlying mechanisms. J Cogn Neurosci 32:1221-1241.

Brainard DH (1997) The Psychophysics Toolbox. Spat Vis 10:433-436.

Breska A, Deouell LY (2014) Automatic bias of temporal expectations following temporally regular input independently of high-level temporal expectation. J Cogn Neurosci 26:1555-1571.

Breska A, Ivry RB (2018) Double dissociation of single-interval and rhythmic temporal prediction in cerebellar degeneration and Parkinson's disease. Proc Natl Acad Sci U S A 115:12283-12288.

Correa Á, Lupiáñez J, Madrid E, Tudela P (2006) Temporal attention enhances early visual processing: a review and new evidence from event-related potentials. Brain Res 1076:116-128.

Coull JT, Nobre AC (1998) Where and when to pay attention: the neural systems for directing attention to spatial locations and to time intervals as revealed by both PET and fMRI. J Neurosci 18:7426-7435.

de la Rosa MD, Sanabria D, Capizzi M, Correa A (2012) Temporal preparation driven by rhythms is resistant to working memory interference. Front Psychol 3:308.

Doherty JR, Rao A, Mesulam MM, Nobre AC (2005) Synergistic effect of combined temporal and spatial expectations on visual attention. J Neurosci 25:8259-8266.

Donner TH, Siegel M, Fries P, Engel AK (2009) Buildup of choice-predictive activity in human motor cortex during perceptual decision making. Curr Biol 19:1581-1585.

Ergenoglu T, Demiralp T, Bayraktaroglu Z, Ergen M, Beydagi H, Uresin Y (2004) Alpha rhythm of the EEG modulates visual detection performance in humans. Cogn Brain Res 20:376-383.

Fischer R, Plessow F, Ruge H (2013) Priming of visual cortex by temporal attention? The effects of temporal predictability on stimulus(-specific) processing in early visual cortical areas. Neuroimage 66:261-269.
Ghose GM, Maunsell JHR (2002) Attentional modulation in visual cortex depends on task timing. Nature 419:616-620.

Gould IC, Rushworth MF, Nobre AC (2011) Indexing the graded allocation of visuospatial attention using anticipatory alpha oscillations. J Neurophysiol 105:1318-1326.

Gould IC, Nobre AC, Wyart V, Rushworth MFS (2012) Effects of decision variables and intraparietal stimulation on sensorimotor oscillatory activity in the human brain. J Neurosci 32:13805-13818.

Griffin IC, Miniussi C, Nobre AC (2002) Multiple mechanisms of selective attention: differential modulation of stimulus processing by attention to space or time. Neuropsychologia 40:2325-2340.

Haegens S, Händel BF, Jensen O (2011) Top-down controlled alpha band activity in somatosensory areas determines behavioral performance in a discrimination task. J Neurosci 31:5197-5204.

Hanslmayr S, Aslan A, Staudigl T, Klimesch W, Herrmann CS, Bäuml KH (2007) Prestimulus oscillations predict visual perception performance between and within subjects. Neuroimage 37:1465-1473.

Heideman SG, Rohenkohl G, Chauvin JJ, Palmer CE, van Ede F, Nobre AC (2018a) Anticipatory neural dynamics of spatial-temporal orienting of attention in younger and older adults. Neuroimage 178:46-56.

Heideman SG, van Ede F, Nobre AC (2018b) Temporal alignment of anticipatory motor cortical beta lateralisation in hidden visual-motor sequences. Eur J Neurosci 48:2684-2612.

Heideman SG, Quinn AJ, Woolrich MW, van Ede F, Nobre AC (2020) Dissecting beta-state changes during timed movement preparation in Parkinson's disease. Prog Neurobiol 184:101731.

Iemi L, Chaumon M, Crouzet SM, Busch NA (2017) Spontaneous neural oscillations bias perception by modulating baseline excitability. J Neurosci 37:807-819.

Janssen P, Shadlen MN (2005) A representation of the hazard rate of elapsed time in macaque area LIP. Nat Neurosci 8:234-241.

Jaramillo S, Zador AM (2011) The auditory cortex mediates the perceptual effects of acoustic temporal expectation. Nat Neurosci 14:246-251.

Jensen O, Mazaheri A (2010) Shaping functional architecture by oscillatory alpha activity: gating by inhibition. Front Hum Neurosci 4:186.

Jones MR, Boltz M (1989) Dynamic attending and responses to time. Psychol Rev 96:459-491.

Kizuk SAD, Mathewson KE (2017) Power and phase of alpha oscillations reveal an interaction between spatial and temporal visual attention. J Cogn Neurosci 29:480-494.

Lakatos P, Karmos G, Mehta AD, Ulbert I, Schroeder CE (2008) Entrainment of neuronal oscillations as a mechanism of attentional selection. Science 320:110-113.

Lange K, Röder B (2006) Orienting attention to points in time improves stimulus processing both within and across modalities. J Cogn Neurosci 18:715-729.

Lange K, Krämer UM, Röder B (2006) Attending points in time and space. Exp Brain Res 173:130-140.

Lima B, Singer W, Neuenschwander S (2011) Gamma responses correlate with temporal expectation in monkey primary visual cortex. J Neurosci 31:15919-15931.

Los SA, Kruijne W, Meeter M (2017) Hazard versus history: temporal preparation is driven by past experience. J Exp Psychol Hum Percept Perform 43:78-88.

Maris E, Oostenveld R (2007) Nonparametric statistical testing of EEG- and MEG-data. J Neurosci Methods 164:177-190.

Mazaheri A, Nieuwenhuis ILC, van Dijk H, Jensen O (2009) Prestimulus alpha and mu activity predicts failure to inhibit motor responses. Hum Brain Mapp 30:1791-1800.

McFarland DJ, Miner LA, Vaughan TM, Wolpaw JR (2000) Mu and beta rhythm topographies during motor imagery and actual movements. Brain Topogr 12:177-186.

Meijer D, te Woerd E, Praamstra P (2016) Timing of beta oscillatory synchronization and temporal prediction of upcoming stimuli. Neuroimage 138:233-241.

Miniussi C, Wilding EL, Coull JT, Nobre AC (1999) Orienting attention in time: modulation of brain potentials. Brain 122:1507-1518.

Morillon B, Schroeder CE, Wyart V, Arnal LH (2016) Temporal prediction in lieu of periodic stimulation. J Neurosci 36:2342-2347.

Nobre AC (2001) Orienting attention to instants in time. Neuropsychologia 39:1317-1328 
Nobre AC, van Ede F (2018) Anticipated moments: temporal structure in attention. Nat Rev Neurosci 19:34-48.

Oostenveld R, Fries P, Maris E, Schoffelen JM (2011) FieldTrip: open source software for advanced analysis of MEG, EEG, and invasive electrophysiological data. Comput Intell Neurosci 2011:156869.

Pfurtscheller G, Berghold A (1989) Patterns of cortical activation during planning of voluntary movement. Electroencephalogr Clin Neurophysiol 72:250-258.

Pomper U, Keil J, Foxe JJ, Senkowski D (2015) Intersensory selective attention and temporal orienting operate in parallel and are instantiated in spatially distinct sensory and motor cortices. Hum Brain Mapp 36:32463259.

Praamstra P, Kourtis D, Kwok HF, Oostenveld R (2006) Neurophysiology of implicit timing in serial choice reaction-time performance. J Neurosci 26:5448-5455

Riehle A, Grün S, Diesmann M, Aertsen A (1997) Spike synchronization and rate modulation differentially involved in motor cortical function. Science 278:1950-1953.

Rihs TA, Michel CM, Thut G (2009) A bias for posterior $\alpha$-band power suppression versus enhancement during shifting versus maintenance of spatial attention. Neuroimage 44:190-199.

Rohenkohl G, Nobre AC (2011) Alpha oscillations related to anticipatory attention follow temporal expectations. J Neurosci 31:14076-14084.

Rohenkohl G, Coull JT, Nobre AC (2011) Behavioural dissociation between exogenous and endogenous temporal orienting of attention. PLoS One 6: e14620.

Rohenkohl G, Cravo AM, Wyart V, Nobre AC (2012) Temporal expectation improves the quality of sensory information. J Neurosci 32:8424-8428.

Rohenkohl G, Gould IC, Pessoa J, Nobre AC (2014) Combining spatial and temporal expectations to improve visual perception. J Vis 14:8, 1-1

Schoffelen JM, Oostenveld R, Fries P (2005) Neuronal coherence as a mechanism of effective corticospinal interaction. Science 308:111-113.

Shalev N, Nobre AC, van Ede F (2019) Time for what? Breaking down temporal anticipation. Trends Neurosci 42:373-374.

Shin JC, Ivry RB (2002) Concurrent learning of temporal and spatial sequences. J Exp Psychol Learn Mem Cogn 28:445-457.
Thut G, Nietzel A, Brandt SA, Pascual-Leone A (2006) $\alpha$-Band electroencephalographic activity over occipital cortex indexes visuospatial attention bias and predicts visual target detection. J Neurosci 26:9494-9502.

Todorovic A, Schoffelen J-M, Van Ede F, Maris E, De Lange FP (2015) Temporal expectation and attention jointly modulate auditory oscillatory activity in the beta band. PLoS One 10:e0120288.

Triviño M, Arnedo M, Lupiáñez J, Chirivella J, Correa A (2011) Rhythms can overcome temporal orienting deficit after right frontal damage. Neuropsychologia 49:3917-3930.

van Dijk H, Schoffelen JM, Oostenveld R, Jensen O (2008) Prestimulus oscillatory activity in the alpha band predicts visual discrimination ability. J Neurosci 28:1816-1823.

van Ede F, de Lange F, Jensen O, Maris E (2011) Orienting attention to an upcoming tactile event involves a spatially and temporally specific modulation of sensorimotor alpha- and beta-band oscillations. J Neurosci 31:2016-2024.

van Ede F, de Lange FP, Maris E (2012a) Attentional cues affect accuracy and reaction time via different cognitive and neural processes. J Neurosci 32:10408-10412.

van Ede F, Köster M, Maris E (2012b) Beyond establishing involvement: quantifying the contribution of anticipatory $\alpha$ - and $\beta$-band suppression to perceptual improvement with attention. J Neurophysiol 108:23522362.

van Ede F, Niklaus M, Nobre AC (2017) Temporal expectations guide dynamic prioritization in visual working memory through attenuated $\alpha$ oscillations. J Neurosci 37:437-445.

van Elswijk G, Kleine BU, Overeem S, Stegeman DF (2007) Expectancy induces dynamic modulation of corticospinal excitability. J Cogn Neurosci 19:121-131.

Vangkilde S, Coull JT, Bundesen C (2012) Great expectations: temporal expectation modulates perceptual processing speed. J Exp Psychol Hum Percept Perform 38:1183-1191.

Worden MS, Foxe JJ, Wang N, Simpson GV (2000) Anticipatory biasing of visuospatial attention indexed by retinotopically specific alpha-band electroencephalography increases over occipital cortex. J Neurosci 20:RC63.

Zanto TP, Pan P, Liu H, Bollinger J, Nobre AC, Gazzaley A (2011) Agerelated changes in orienting attention in time. J Neurosci 31:1246112470 